\title{
| COMMENTAIRE DU CEA
}

\author{
Jean-Louis Boutard
}

Après le premier chapitre de présentation de la Métallurgie comme science en liaison forte avec les applications, ce rapport établit un diagnostic de la situation pour divers secteurs industriels et pour la recherche et l'enseignement.

D'une manière générale il s'agit d'un rapport bien documenté, dont la lecture n'est cependant pas très facile compte tenu de ce parti pris d'entrer par application, qui conduit à un éparpillement certain.

\section{Un premier chapitre dédié uniquement aux disciplines scientifiques de la métallurgie}

Le contenu disciplinaire de la métallurgie se trouve traité en partie dans le premier chapitre et dans l'annexe 25 sur l'enseignement. Néanmoins dans ce premier chapitre, le contenu disciplinaire de la métallurgie d'élaboration pourtant très riche est pratiquement absent, au profit $d$ 'autres considérations économiques ou écologiques. II faut aller chercher l'analyse des disciplines scientifiques de la métallurgie d'élaboration en annexe 25.

Le rapport gagnerait en clarté à présenter dans un premier chapitre le contenu des disciplines scientifiques de la métallurgie, à l'exclusion de toutes autres considérations traitées dans les chapitres applicatifs suivants. II serait souhaitable qu'il intègre aussi un résumé de l'état des lieux de l'enseignement actuel. Cela me paraît être une base indispensable pour les mesures à prendre en ce qui concerne l'enseignement.

Concernant le résumé et recommandations, trois remarques ci-après.

Les deux premières portent sur le chapeau de ce résumé où manquent l'aspect vieillissement en service et la citation du CEA (et de l'Onera) comme organismes de recherche dédiée où le nombre de métallurgistes a fortement décru comme indiquée dans le corps du texte. 


\section{Résumé et recommandations : le vieillissement des matériaux...}

La première phrase du résumé présente la métallurgie comme "science des métaux et discipline technique liée à la production, la mise en forme et l'assemblage des métaux ". II manque la maîtrise du vieillissement et des propriétés d'usage, qui est un moteur essentiel de la métallurgie appliquée et de base, par exemple pour le nucléaire au CEA et l'aéronautique à l'Onera.

Nous proposons que cet aspect de vieillissement et maîtrise des propriétés d'usage soit ajouté dans la première phrase du résumé.

\section{Résumé et recommandations : les grands utilisateurs (transport, énergie) perdent leurs experts, tout comme les centres techniques...}

Cette affirmation est incomplète. Le rapport souligne aussi à juste titre que les organismes dédiés, CEA et Onera, ont vu leurs effectifs de chercheurs métallurgistes divisés par deux dans les vingt dernières années. II me paraît indispensable de le rappeler dans le résumé. Donc « les grands utilisateurs (transport, énergie) et les organismes dédiés (CEA, Onera) perdent leurs experts tout comme les centres techniques... »

Suivent ensuite des recommandations pour une Mission interministérielle chargée de redéfinir et mettre en œuvre une nouvelle politique industrielle et de recherche-enseignement, en liaison étroite.

La recommandation 3 concerne les organismes de recherche dédiée. Elle recommande une meilleure imbrication des aspects appliquées et fondamentaux. Cette affirmation ne nous paraît pas adéquate. 


\section{Résumé et recommandations : « Recommandations 3 : que science et génie métallurgiques soient individualisés par les grands organismes de recherche comme activités idéalement interdisciplinaires, mêlant au mieux l'appliqué et le fondamental. »}

Il faut être plus clair et demander le maintien d'un financement public gouvernemental pour permettre au CEA et à l'Onera de mener les recherches de métallurgie de base et appliquée nécessaires aux défis de l'industrie aéronautique et au développement des réacteurs de génération IV.

Il s'agit en effet de deux domaines où on doit développer des matériaux devant résister à des conditions en service difficiles (haute température et irradiation), et, prédire de manière fiable l'évolution des propriétés d'usage à partir de l'échelle atomique où diffusion et effets d'irradiation peuvent être décrits de manière sûre ou du moins avec des hypothèses maîtrisées, une telle prédiction nécessitant donc une simulation numérique multi-échelles.

CEA et Onera ayant développé une recherche fondamentale et appliquée reconnue au plan national et international devraient constituer des points d'appui pour un enseignement de métallurgie de qualité.

Nous proposons donc de reformuler cette recommandation 3 comme suit :

"Que la recherche métallurgique de base et appliquée conduite, dans des organismes dédiés comme le CEA et l'Onera, continue d'être financée sur fonds publics gouvernementaux pour répondre aux défis de l'industrie nucléaire et aéronautique, et, constituer des points d'appui indispensables à un enseignement de qualité. » 


\section{KVSDJHIQQMQWRQDQ}

